\section{Hesitação vacinal para influenza entre trabalhadores(as) da saúde, Bahia, Brasil}

\author{
Influenza vaccine hesitancy among health \\ workers, Bahia State, Brazil
}

\section{Vacilación en la vacunación contra la gripe entre trabajadores/as de salud, Bahía, Brasil}

Fernanda de Oliveira Souza 1,2

Guilherme Loureiro Werneck 3

Paloma de Sousa Pinho 1

Jules Ramon Brito Teixeira 2

Iracema Lua 4

Tânia Maria de Araújo 2

\section{Resumo}

A Organização Mundial da Saúde reconhece a hesitação vacinal como uma das dez maiores ameaças à saúde pública no mundo. Este estudo investigou a associação entre confiança, conveniência e complacência e a hesitação vacinal para influenza entre trabalhadores(as) do setor saúde. Foram incluídos 453 trabalhadores(as) de serviços de atenção primária e média complexidade de uma cidade de médio porte do estado brasileiro da Bahia. Foram considerados hesitantes em vacinar aqueles que não receberam vacina para influenza em 2019. Modelos de equações estruturais foram utilizados para avaliar interrelações entre variáveis preditoras de interesse e hesitação vacinal. Um quarto dos(as) trabalhadores(as) $(25,4 \%)$ hesitaram em se vacinar para influenza. Menor confiança (coeficiente padronizado $-C P=0,261 ; p=0,044$ ) e maior complacência $(C P=0,256 ; p<0,001)$ associaram-se significativamente à hesitação vacinal. A conveniência não esteve associada à hesitação vacinal. Trabalhadores(as) não assistenciais, da média complexidade e do sexo masculino referiram menor acolhimento pelo profissional que administrava as vacinas. O medo de agulhas associou-se à menor confiança e à hesitação vacinal. História de reação vacinal não esteve associada diretamente com hesitação vacinal, mas associou-se à maior complacência, isto é, menor percepção do risco de doenças imunopreveníveis. Por ser uma vacina recomendada anualmente, a hesitação vacinal para influenza pode contribuir para aumentar a carga da doença na população. Os dados sustentam a hipótese de que fatores relacionados à confiança e complacência produzem prejuízos na aceitação desta vacina, devendo ser considerados no desenvolvimento de estratégias e ações para maior adesão à vacinação.

Vacinação; Trabalhadores da Saúde; Recusa de Vacinação

\author{
Correspondência \\ F. O. Souza \\ Rua Bartolomeu de Gusmão s/n, Feira de Santana, BA \\ 44021-165, Brasil. \\ nandaolisouza@gmail.com \\ 1 Universidade Federal do Recôncavo da Bahia, Feira de \\ Santana, Brasil. \\ 2 Universidade Estadual de Feira de Santana, Feira de Santana, \\ Brasil. \\ 3 Instituto de Medicina Social, Universidade do Estado do Rio de \\ Janeiro, Rio de Janeiro, Brasil. \\ 4 Instituto de Saúde Coletiva, Universidade Federal da Bahia, \\ Salvador, Brasil.
}




\section{Introdução}

A influenza é doença respiratória viral aguda de alta transmissibilidade e ampla distribuição global. O vírus provoca epidemias sazonais e, eventualmente, pandemias, quando mudanças antigênicas maiores fazem surgir uma nova variante do vírus, para a qual a população não apresenta imunidade 1,2 . No mundo, as epidemias sazonais de gripe geram aproximadamente cinco milhões de casos de doença grave e até 650 mil mortes anuais 3 .

A gripe sazonal tem grande impacto na saúde pública 4,5 e, entre as medidas disponíveis para prevenir a doença e os seus desdobramentos graves, a vacinação é a mais efetiva 6,7. A maioria dos países latino-americanos mantêm uma política de vacinação contra influenza sazonal em vigor 8 . O Ministério da Saúde 2 recomenda a vacinação de grupos de risco, incluindo os trabalhadores(as) da saúde.

Os trabalhadores(as) da saúde têm risco adicional de serem infectados com influenza quando comparados à população em geral, principalmente em função do frequente contato com pessoas infectadas. No Brasil, a vacinação contra os tipos A e B do vírus influenza é disponibilizada de forma gratuita mediante campanha anual de vacinação 2 .

Apesar da existência de programas de vacinação para trabalhadores(as) da saúde em muitos países, a cobertura vacinal para influenza não atinge as metas desejáveis. Na Arábia Saudita, o percentual de trabalhadores vacinados contra gripe de seis grandes hospitais foi de apenas $38 \%{ }^{9}$. Nos países europeus, a cobertura da vacinação contra influenza sazonal entre profissionais de saúde foi de 49,7\% na Espanha 10, 60\% na Alemanha 11 e variou de 16,7 a 30,6\% na Itália 12,13. Estudo com 755 trabalhadores da saúde de um serviço hospitalar no Brasil identificou cobertura global da vacina contra influenza de $61,5 \%$, sendo maior entre enfermeiros (69\%) e menor entre médicos (49,1\%) 14 .

A hesitação vacinal se refere ao atraso na aceitação ou recusa da vacinação, apesar da disponibilidade nos serviços 15. Embora o problema seja um fenômeno de difícil abordagem em qualquer contexto, a sua análise entre trabalhadores da saúde é ainda mais complexa, considerando que: o trabalho cotidiano envolve risco real de infecção, o acesso pode ser maior devido à disponibilidade da vacina no serviço de saúde e, em geral, há um nível mais elevado de conhecimento dos riscos e benefícios da vacinação.

A complexidade do assunto tem envolvido múltiplos investimentos na sua compreensão. O Grupo Consultivo Estratégico de Especialistas em Imunização (Strategic Advisory Group of Experts on Immunization - SAGE) da Organização Mundial da Saúde (OMS) propôs um modelo de análise da hesitação vacinal denominado "3Cs", que remete a três determinantes: Confiança, Conveniência e Complacência. A confiança relaciona-se ao conhecimento e a percepções sobre segurança e eficácia das vacinas, considerando as experiências anteriores de reações adversas, e ao crédito atribuído a instituições, serviços e profissionais de saúde envolvidos no processo de vacinação. A conveniência envolve a disponibilidade, acessibilidade geográfica dos serviços de vacinação, acesso à informação e capacidade de compreensão. A complacência diz respeito à baixa percepção individual do risco de doenças imunopreveníveis e, consequentemente, do valor atribuído às vacinas 15 . Este modelo de avaliação da hesitação vacinal vem sendo aprimorado e, recentemente, foi sugerida a escala "5C" que acrescenta dois novos antecedentes psicológicos para decisão de vacinar 16.

Os instrumentos de pesquisa têm sido desenvolvidos e utilizados para avaliar hesitação vacinal na infância e adolescência 17 . Os estudos que examinaram as barreiras relacionadas à vacinação contra gripe entre profissionais de saúde limitaram-se aos profissionais de serviços hospitalares 6,13,18,19,20,21. Em situações dramáticas, como o atual contexto pandêmico, a vacinação se torna elemento central na prática de saúde pública para contenção e propagação da infecção, mas, para isso, a cobertura vacinal adequada precisa ser alcançada 22.

Em 2019, a OMS reconheceu a hesitação vacinal como uma das dez maiores ameaças à saúde pública no mundo, destacando que fatores como falta de confiança, conveniência e complacência são elementos subjacentes a esse fenômeno ${ }^{23}$. Segundo a OMS, trabalhadores(as) da saúde são os principais influenciadores nas decisões sobre vacinação e devem ser apoiados para fornecer informações confiáveis 23 . Assim, compreender o problema da hesitação em relação à vacinação contra influenza entre trabalhadores(as) da saúde poderá orientar ações para ampliação da vacinação e produzir conhecimento com potencial para ser empregado na abordagem de outros agravos imunopreveníveis 24 . 
O foco de análise em trabalhadores(as) da saúde justifica-se em função desses profissionais desempenharem papel crucial na promoção da aceitação de vacinas, além de estarem sob risco para infecção 25 .

Este estudo objetivou investigar a associação entre confiança, complacência e conveniência com a hesitação vacinal para influenza entre trabalhadores(as) do setor saúde.

\section{Métodos}

Este estudo é parte da pesquisa Vigilância e Monitoramento para Doenças Infecciosas entre Trabalhadores e Trabalhadoras do Setor Saúde. Informações mais detalhadas deste estudo podem ser encontrados em http://saudedostrabalhadoresdasaude.uefs.br.

\section{Participantes e amostra}

Trata-se de um estudo transversal entre trabalhadores(as) da rede de saúde de um município de médio porte da Bahia, Brasil. Foram incluídos os trabalhadores(as) em efetivo exercício profissional dos serviços de saúde da atenção primária e da média complexidade. A coleta de dados foi realizada no período de junho a dezembro de 2019.

Foram considerados como profissionais assistenciais os trabalhadores com formação específica de nível técnico ou superior (médicos, enfermeiras, odontólogos, fisioterapeutas, assistentes sociais, nutricionistas, farmacêuticos, psicólogos, terapeutas ocupacionais, técnicos em odontologia e em enfermagem). A categoria de apoio técnico, operacional e administrativa foi formada por trabalhadores sem formação (graduação ou curso técnico) específica em saúde: agentes de saúde, pessoal técnico-administrativo (recepcionistas/atendentes de farmácia), seguranças, motoristas e serviços gerais.

Para o cálculo amostral, considerou-se a população total de trabalhadores(as) dos serviços de saúde da atenção básica e de média complexidade do município (622), prevalência de 61,5\% de vacinação incompleta no calendário vacinal do adulto 26 , erro de $3 \%$, nível de $95 \%$ de confiança e acréscimo de $20 \%$ para possíveis perdas e/ou recusas. Estimou-se amostra de 410 trabalhadores.

Adotou-se amostragem aleatória estratificada por nível de complexidade do serviço e por grupos ocupacionais. Realizou-se levantamento prévio do número de trabalhadores(as) segundo os estratos de nível de complexidade dos serviços e grupo ocupacional. Definido o tamanho amostral, procedeuse ao cálculo da participação percentual segundo os estratos. Em seguida, sortearam-se, com auxílio de lista de números aleatórios, os(as) trabalhadores(as) a serem estudados(as). Os(as) trabalhadores(as) foram contatados(as) e entrevistados(as) nos locais de trabalho.

\section{Coleta de dados e variáveis do estudo}

Utilizou-se questionário previamente testado, estruturado em blocos de questões: sexo (feminino/ masculino), faixa etária (21 a 30 anos/31 a 49 anos/mais de 50 anos); escolaridade (até Ensino Médio/ Técnico e Superior), raça/cor (pretos/não pretos), renda (até dois salários mínimos/mais de dois salários); ocupacionais: tempo de trabalho (até cinco anos/mais de cinco anos), categoria profissional (assistencial/apoio técnico e operacionais), vínculo de trabalho (temporário/permanente), nível de complexidade (atenção primária/média complexidade), história de reação vacinal (sim/não), medo no momento da aplicação (sim/não), acolhimento pelo profissional responsável pela vacinação (sim/não). $\mathrm{O}$ ponto de corte de dois salários mínimos foi definido tomando como base a mediana de $\mathrm{R} \$ 1.800$, valor próximo a dois salários mínimos (em 2019 - R\$ 998,00).

O desfecho do estudo (hesitação vacinal) foi obtido a partir da resposta à pergunta: "Você se vacinou no último ano contra a influenza?". Foram considerados hesitantes aqueles que responderam "não"; ou seja, que não receberam dose da vacina em 201927.

As dimensões dos 3Cs estão fundamentadas teoricamente no modelo da OMS 15,27. O bloco utilizado para explorar a hesitação em relação às vacinas foi construído com base em revisão da literatura, na estrutura do modelo 3Cs e no instrumento de Blue \& Valley (2002), adaptado por Shahrabani et al. (2009) 28,29 , traduzido e adaptado para o português por Neves et al. (2020) 30, em estudo baseado no Modelo de Crenças em Saúde. 
As variáveis confiança, complacência e conveniência foram operacionalizadas como variáveis latentes, valendo-se de variáveis manifestas (diretamente observadas) (Modelo 1). Confiança foi composta por variáveis de resposta dicotômicas ( $\operatorname{sim} /$ não) referentes às seguintes questões (Tabela 1): já aconteceu algo na sua vida ou comunidade que fez com que você deixasse de acreditar nas vacinas (C1); acredita que o governo fornece a melhor vacina (C2); confia em informações sobre vacinas (C3); tem segurança para receber novas vacinas (C4) e confia nos profissionais que cuidam do processo de vacinação (C5). O item C1 apresenta direção oposta à confiança, ou seja, os indivíduos que responderam positivamente a esse item apresentaram menor confiança na vacina. Nos itens C2 a C5, as respostas positivas correspondem a maior confiança. Para análise, os itens C2 a C5 foram revertidos de forma que se tornassem compatíveis com o item C1, isto é, respostas positivas indicando menor confiança e, portanto, maior hesitação vacinal.

As respostas para as questões sobre conveniência são dicotômicas (sim/não): acesso a informações suficientes para decidir se vacinar (V1); recebe informações sobre riscos/benefícios (V2); acesso à campanha de vacinação no trabalho (V3) e acesso às informações sobre vacinação nos meios de comunicação (V4). Respostas positivas de V1 a V4 indicam maior conveniência para vacinar. Para fins de análise (ver subseção Análise Estatística), os itens foram revertidos de forma que respostas positivas indicam menor conveniência.

A operacionalização da variável latente complacência se baseou em três variáveis observadas, com duas categorias de resposta (concordo/discordo) (Tabela 1): trabalhar com muitas pessoas todo dia aumenta minhas chances de pegar gripe (L1); percebe vantagem em receber vacina da gripe (L2); para me vacinar contra gripe, eu precisaria abrir mão de minhas concepções sobre utilidade, benefícios e riscos da vacinação (L3). Respostas "discordo" para L1 e L2 indicam maior complacência, portanto maior disposição a hesitar na vacinação. O item L3 apresenta direção oposta à complacência, ou seja, os indivíduos que responderam "discordo" a esse item apresentaram menor complacência, isto é,

Tabela 1

Distribuição dos itens que representam os 3Cs do modelo de hesitação vacinal. Bahia, Brasil, 2020.

\begin{tabular}{|c|c|c|c|}
\hline Itens & $\mathbf{n}$ & $\%$ & IC95\% \\
\hline \multicolumn{4}{|l|}{ Confiança (resposta: Sim) * } \\
\hline C1. Já aconteceu algo na sua vida ou comunidade que fez com que você deixasse de acreditar nas vacinas? & 32 & 7,1 & $4,9-9,8$ \\
\hline C2. Você acredita que o governo fornece a melhor vacina do mercado? & 293 & 70,6 & $65,9-74,9$ \\
\hline C3. Você confia em informações que os profissionais lhe repassam sobre vacinação? & 406 & 90,2 & $87,1-92,8$ \\
\hline C4. Você se sente seguro para receber novas vacinas? & 367 & 81,4 & $77,4-84,8$ \\
\hline C5. Você confia nas pessoas que cuidam do processo de vacinação na Unidade Saúde da Família? & 414 & 91,8 & $88,8-94,1$ \\
\hline \multicolumn{4}{|l|}{ Conveniência (resposta: Sim) * } \\
\hline V1. Nas campanhas de vacinação, você tem informações suficientes para decidir se vacinar? & 367 & 81,6 & $77,6-85,0$ \\
\hline V2. Você se sente informado suficiente sobre riscos/benefícios da vacinação? & 350 & 77,3 & $73,1-81,0$ \\
\hline V3. Eu me vacinei contra gripe porque foi realizada campanha de vacinação no meu trabalho. & 250 & 55,8 & $51,0-60,4$ \\
\hline $\begin{array}{l}\text { V4. Eu me vacinei contra gripe após ouvir informações sobre os benefícios da vacina nos meios de } \\
\text { comunicação (televisão, rádio, redes sociais). }\end{array}$ & 246 & 54,9 & $50,1-59,5$ \\
\hline \multicolumn{4}{|l|}{ Complacência (resposta: Discordo) * } \\
\hline L1. Trabalhar com muitas pessoas todo dia aumenta minhas chances de pegar gripe. & 63 & 14,0 & $10,9-17,5$ \\
\hline L2. Eu tenho muito a ganhar ao me vacinar contra gripe. & 79 & 18,1 & $14,5-22,0$ \\
\hline $\begin{array}{l}\text { L3. Para me vacinar contra gripe, eu precisaria abrir mão de minhas concepções sobre utilidade, benefícios } \\
\text { e riscos da vacinação. }\end{array}$ & 367 & 81,6 & $77,6-85,0$ \\
\hline
\end{tabular}

IC95\%: intervalo de 95\% de confiança.

* Confiança: respostas "Sim" indicam maior confiança, exceto para C1. Conveniência: respostas "Sim" indicam maior conveniência. Complacência: respostas "Discordo" indicam menor complacência, exceto para L3. Nas análises os itens C2 a C5, V1 a V4 e L1 e L2, foram revertidos para a composição de escores de confiança, conveniência e complacência cuja pontuação foi ordenada de modo a evidenciar aumento na hesitação vacinal (menor confiança, menor conveniência e maior complacência). 
estariam mais dispostos a vacinar. Para fins de análise (ver subseção Análise Estatística), o item L3 foi revertido de forma que se tornasse compatível com os itens L1 e L2, isto é, respostas positivas indicando maior complacência e, consequentemente, maior propensão à hesitação vacinal.

\section{Análise estatística}

Foi processada análise descritiva das características sociodemográficas e ocupacionais dos participantes. A análise confirmatória utilizando modelos de equações estruturais (SEM) foi conduzida com o intuito de avaliar interrelações entre variáveis preditoras de interesse e a hesitação vacinal 31,32 .

O SEM é composto pelo modelo de mensuração e pelo modelo estrutural 33,34 . O modelo de mensuração foi proposto a partir de análise fatorial de cada construto proposto pelo modelo 3Cs (confiança, complacência e conveniência). Cada construto latente (variável não observada diretamente) foi inicialmente operacionalizado com, no mínimo, três itens (variáveis observadas), como recomendado 35.

Inicialmente, especificou-se o modelo de equação estrutural exploratório (MEEE) 36 com uso dos itens disponíveis. Análise fatorial confirmatória (AFC) realizada para avaliar a validade do modelo do construto latente. Avaliou-se o carregamento dos itens, tendo como critérios a carga fatorial estandardizada $\geq 0,3$ e variância residual $\leq 0,5$ 37,38. Independentemente da variância, itens com carga fatorial padronizada inferiores a 0,3 foram excluídos.

O modelo estrutural inicialmente testado foi composto por estruturas de associação teóricas (caminhos causais) entre as variáveis de interesse e cada componente do modelo 3Cs separadamente (Figura 1). Foram avaliados caminhos causais diretos ou indiretos. Os caminhos diretos foram aqueles que ligavam a variável diretamente à hesitação vacinal. Os caminhos indiretos foram aqueles interceptados por uma terceira variável, considerada mediadora (Figura 1).

Para as análises estatísticas, a pontuação das categorias das variáveis foi ordenada de modo a evidenciar aumento na hesitação vacinal para influenza (categorias de exposição): (1) menor confiança; (2) menor conveniência; (3) maior complacência; (4) maior medo; (5) menor acolhimento; (6) sexo masculino; (7) fazer parte da categoria de apoio técnico, operacional e administrativa; (8) trabalhar na média complexidade; e (9) ter história de reação vacinal. O tamanho do efeito (coeficiente padronizado - CP) foi classificado em: pequeno (em torno de 0,1), médio (próximo a 0,3) e forte/grande $(>0,5) 39$.

O estimador de mínimos quadrados ponderados robusto (WLSMV) foi empregado para estimar os coeficientes de regressão brutos e padronizados, com intervalos de $95 \%$ de confiança (IC95\%) e valor de $\mathrm{p}<0,05$. Para inspecionar as correlações residuais entre os itens e a necessidade de reespecificação do modelo avaliaram-se os índices de modificação (MI) e respectivas mudanças esperadas de parâmetros (MEP). Valores de MI $\geq 10$ e MEP $\geq 0,25$ indicaram a necessidade de avaliação detalhada de possibilidades de reespecificação do modelo 37,38,40. Para o modelo de SEM final foram mantidos os caminhos causais estatisticamente significantes e aqueles que contribuíram para o melhor ajuste de confundimento, evidenciado pela avaliação da adequação dos índices de ajuste do modelo.

Os índices de ajuste comparativo (CFI) e de Tucker-Lewis (TLI) e a raiz do erro médio quadrático de aproximação (RMSEA) foram utilizados para avaliar os modelos. Os CFI e TLI variam de 0 a 1 e valores acima de 0,9 indicam um bom ajuste. Para RMSEA, valores $<0,06$ sugerem bom ajuste, e os valores $>0,1$ indicam ajuste ruim e a rejeição do modelo 37,40.

As análises descritivas foram realizadas por recurso do SPSS, versão 22 (https://www.ibm.com/), e a análise SEM processada por meio do software estatístico Mplus, versão 8.4 (https://www.statmo del.com/).

\section{Aspectos éticos}

O estudo foi aprovado pelo Comitê de Ética em Pesquisa da Universidade Estadual de Feira de Santana (parecer no 2.897062). Os participantes da pesquisa assinaram o Termo de Consentimento Livre e Esclarecido de acordo com os princípios éticos da Resolução no 466/2012 e da Resolução no 510/2016. 


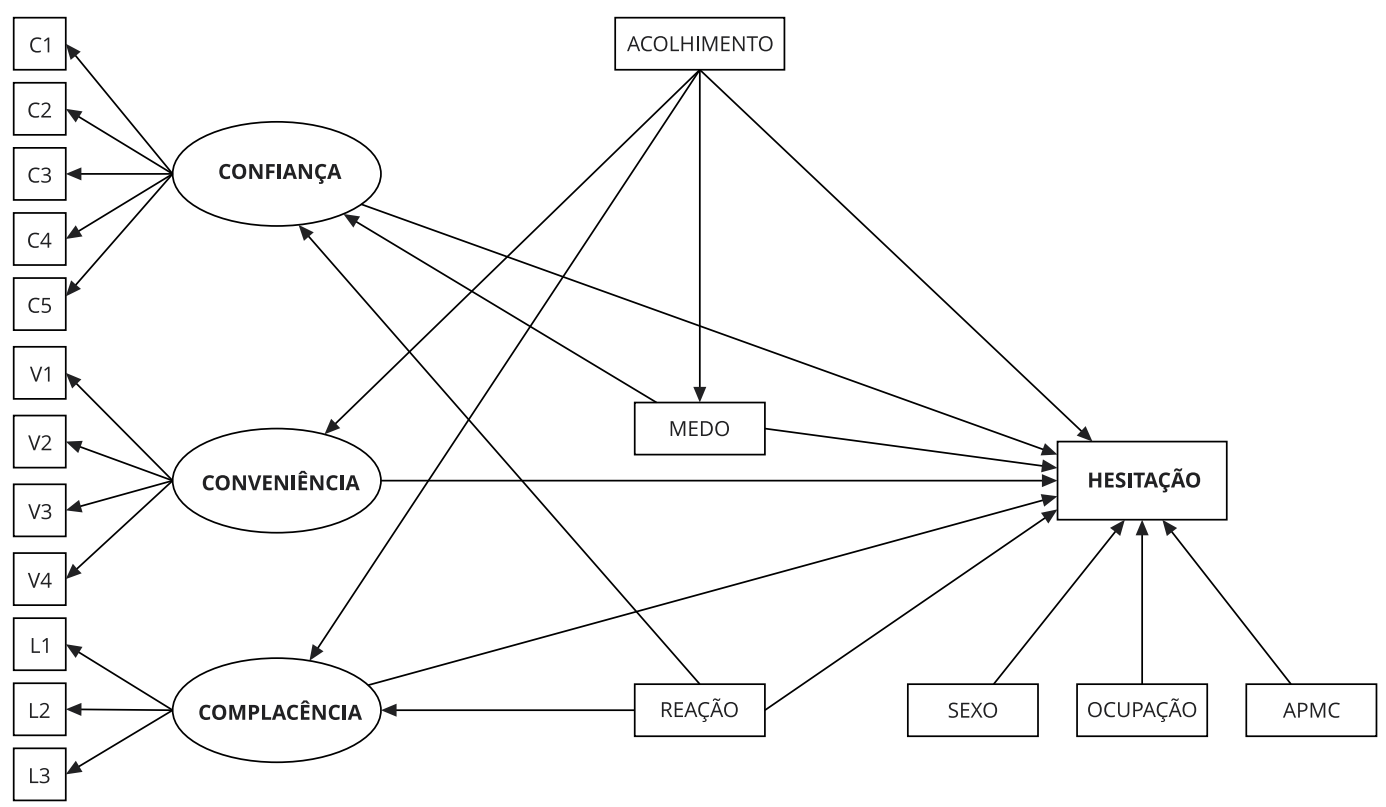

APMC: atenção primária e média complexidade.

\section{Resultados}

Participaram deste estudo 453 trabalhadores(as) da saúde, com predominância das mulheres (82,8\%), com idade variando entre 21 e 72 anos e média de idade de 42,3 anos (desvio padrão - DP =9,4) (Tabela 2). Um quarto $(25,4 \%)$ hesitaram em se vacinar para influenza.

Inicialmente foi estimado o modelo de mensuração com o uso dos itens propostos para mapear os 3Cs (confiança, complacência e conveniência) do modelo 3Cs (Tabela 1).

Guiados pelas dimensões teoricamente propostas, procedeu-se a análise do modelo de equação estrutural exploratório para cada uma delas, conduzindo-se uma série de AFC para avaliar o modelo de mensuração para os 3Cs. Desse modo, foram obtidos três modelos estruturais exploratórios, testados de modo subsequente (Tabela 3):

Modelo 1: as variáveis relacionadas à confiança obtiveram cargas fatoriais e índices de ajuste satisfatórios, indicando a convergência em uma variável latente. Para complacência, a vantagem em se vacinar (L2) obteve elevada carga fatorial $(0,935)$, evidenciando que apenas ela seria suficiente para representar essa dimensão. Para conveniência, foi identificada alta correlação entre V3 e V4 e baixa carga fatorial para V3, indicando a necessidade de exclusão de V3;

Modelo 2: as variáveis relacionadas à confiança mantiveram-se com cargas fatoriais satisfatórias. Com relação à conveniência, a carga fatorial de V4 foi inferior a 0,3 , indicando a sua exclusão. Dois itens mantiveram-se no modelo (V1 e V2) e, considerando que são necessários, no mínimo, três itens para composição do construto latente, optou-se por analisar a conveniência como variável diretamente observada. Construiu-se um indicador considerando como "sim" se o participante respondeu positivamente a V1 e/ou V2 e como "não" quando negativou os dois itens; 
Tabela 2

Características sociodemográficas e ocupacionais dos trabalhadores da atenção primária e média complexidade de saúde e prevalência de hesitação vacinal para influenza. Bahia, Brasil, 2020.

\begin{tabular}{|c|c|c|c|c|c|}
\hline \multirow[t]{2}{*}{ Variáveis * } & \multicolumn{2}{|c|}{ Total } & \multicolumn{2}{|c|}{$\begin{array}{c}\text { Prevalência de hesitação } \\
\text { vacinal }\end{array}$} & \multirow[t]{2}{*}{ IC95\% } \\
\hline & $\mathbf{n}$ & $\%$ & $\mathbf{n}$ & $\%$ & \\
\hline Total & 453 & 100,0 & 114 & 25,4 & $21,2-29,4$ \\
\hline \multicolumn{6}{|l|}{ Sociodemográficas } \\
\hline \multicolumn{6}{|l|}{ Sexo $(n=453)$} \\
\hline Feminino & 375 & 82,8 & 88 & 23,7 & $19,2-28$ \\
\hline Masculino & 78 & 17,2 & 26 & 33,8 & $23,0-44,9$ \\
\hline \multicolumn{6}{|l|}{ Escolaridade $(n=440)$} \\
\hline Ensino Fundamental & 13 & 3,0 & 4 & 30,8 & $9,1-61,4$ \\
\hline Ensino Médio & 120 & 27,3 & 34 & 28,3 & $20,5-37,3$ \\
\hline Ensino Técnico & 87 & 19,8 & 19 & 22,1 & $13,7-32,0$ \\
\hline Ensino Superior & 142 & 32,3 & 43 & 30,9 & $22,8-38,5$ \\
\hline Pós-graduação & 78 & 17,7 & 11 & 14,1 & $7,2-23,8$ \\
\hline \multicolumn{6}{|l|}{ Faixa etária (anos) $(n=442)$} \\
\hline $21-30$ & 44 & 10,0 & 10 & 23,3 & $11,4-37,8$ \\
\hline $31-49$ & 292 & 66,1 & 73 & 25,3 & $20,1-30,3$ \\
\hline Mais de 50 & 106 & 24,0 & 27 & 25,5 & $17,5-34,8$ \\
\hline \multicolumn{6}{|c|}{ Renda familiar mensal (salários mínimos) $(n=374)$} \\
\hline$\leq 2$ (até $\mathrm{R} \$ 1.996)$ & 253 & 67,6 & 74 & 29,4 & $23,7-35,2$ \\
\hline > $2(\mathrm{R} \$ 1.996$ ou mais $)$ & 121 & 32,4 & 22 & 18,2 & $11,7-26,2$ \\
\hline \multicolumn{6}{|l|}{ Situação conjugal $(n=450)$} \\
\hline Com companheiro(a) & 272 & 60,9 & 69 & 25,7 & $20,3-30,9$ \\
\hline Sem companheiro(a) & 175 & 39,1 & 44 & 25,3 & $18,9-32,2$ \\
\hline \multicolumn{6}{|l|}{ Filhos $(n=444)$} \\
\hline Sim & 328 & 73,9 & 82 & 25,2 & $20,4-30,0$ \\
\hline Não & 116 & 26,1 & 28 & 24,3 & $16,6-32,9$ \\
\hline \multicolumn{6}{|l|}{ Raça/Cor $(n=448)$} \\
\hline Pretos & 146 & 32,9 & 39 & 26,9 & $19,7-34,6$ \\
\hline Pardos & 222 & 50,0 & 51 & 23,2 & $17,6-29,0$ \\
\hline Não negros & 72 & 16,2 & 21 & 29,6 & $19,0-41,0$ \\
\hline Não sabe & 4 & 0,9 & 3 & 75,0 & $19,4-99,3$ \\
\hline \multicolumn{6}{|l|}{ Ocupacionais } \\
\hline \multicolumn{6}{|l|}{ Tempo de trabalho (anos) $(n=453)$} \\
\hline Até 10 & 244 & 53,9 & 51 & 21,8 & $15,9-26,5$ \\
\hline Mais de 10 & 209 & 46,1 & 56 & 26,9 & $20,9-33,3$ \\
\hline \multicolumn{6}{|l|}{ Categoria profissional $(n=444)$} \\
\hline Assistenciais & 137 & 30,9 & 28 & 20,7 & $14,0-28,1$ \\
\hline Apoio técnico & 175 & 39,4 & 55 & 31,4 & $24,6-38,8$ \\
\hline Administrativo e operacional & 132 & 29,7 & 30 & 23,1 & $15,9-30,8$ \\
\hline \multicolumn{6}{|l|}{ Vínculo de trabalho $(n=446)$} \\
\hline Permanente & 308 & 69,1 & 82 & 26,7 & $21,7-31,9$ \\
\hline Temporário & 138 & 30,9 & 31 & 23,0 & $15,8-30,3$ \\
\hline \multicolumn{6}{|l|}{ Complexidade do serviço $(n=453)$} \\
\hline Atenção básica & 352 & 77,7 & 83 & 23,8 & $19,2-28,3$ \\
\hline Média complexidade & 101 & 22,3 & 31 & 31,0 & $21,9-40,6$ \\
\hline
\end{tabular}

IC9\%\%: intervalo de 95\% de confiança.

* Números variam devido a valores faltantes. 
Tabela 3

Indicadores de ajuste dos modelos de mensuração e modelo estrutural, utilizando hesitação vacinal como variável resposta. Bahia, Brasil, 2020.

\begin{tabular}{|c|c|c|c|c|}
\hline Índices & Modelo 1 * & Modelo 2 * & Modelo 3 * & Modelo estrutural \\
\hline \multicolumn{5}{|l|}{ RMSEA } \\
\hline Índice & 0,046 & 0,035 & 0,055 & 0,023 \\
\hline IC90\% & $0,032-0,059$ & $0,000-0,059$ & 0,012-0,096 & $0,000-0,039$ \\
\hline $\mathrm{CFI}$ & 0,953 & 0,985 & 0,985 & 0,974 \\
\hline TLI & 0,938 & 0,979 & 0,968 & 0,964 \\
\hline
\end{tabular}

CFI: índice de ajustamento comparativo; IC90\%: intervalo de 90\% de confiança; RMSEA: raiz do erro quadrático médio de aproximação; TLI: índice Tucker-Lewis.

* Modelos de mensuração 1, 2 e 3.

Modelo 3: apenas a dimensão confiança foi mensurada a partir de uma variável latente, com cargas fatoriais satisfatórias em todos itens e bom ajuste (Tabela 3). O item que apresentou maior carga foi o C5 ("Você confia em informações que os profissionais lhe repassam sobre vacinação?") e a menor foi C2 (“Já aconteceu algo na sua vida ou comunidade que fez com que você deixasse de acreditar nas vacinas?”) (Tabela 4). As dimensões de conveniência (V1+V2) e complacência (L2) foram analisadas no modelo estrutural como variáveis diretamente observadas.

O modelo estrutural foi constituído pela variável latente confiança, as variáveis observadas complacência e conveniência, avaliando-se a associação direta desses fatores com hesitação vacinal, bem como as relações estruturais entre as outras variáveis propostas no modelo teórico e os 3Cs entre si (Figura 2). O SEM estimado apresentou ajuste adequado (Tabela 3), com relações diretas e indiretas entre as variáveis incluídas.

As soluções fatoriais para variável latente confiança foram significativas $(p<0,001)$. A variável latente confiança $(C P=0,261 ; p=0,044)$ e a variável observada complacência $(C P=0,256 ; p<0,001)$, com tamanho de efeito médio, associaram-se significativamente à hesitação vacinal, revelando que trabalhadores(as) com menor confiança e maior complacência são mais hesitantes para vacinação contra influenza.

O efeito direto de acolhimento para hesitação vacinal apresentou carga baixa, não significante e piora dos índices de ajuste do modelo, indicando a necessidade de exclusão do caminho direto e avaliação do efeito indireto. Analisando o efeito indireto, verificou-se que o acolhimento pelo(a) profissional responsável pela vacinação apresentou efeito positivo e estatisticamente significativo sobre a confiança, conveniência e complacência, sugerindo que, quanto menor o acolhimento, menor confiança e conveniência e maior complacência entre os(as) trabalhadores(as). A exclusão de qualquer um dos caminhos indiretos apresentados na Figura 2 não repercutiu em modificação dos efeitos no modelo estrutural, mas implicou piora dos índices de ajuste, justificando a permanência do caminho indireto (sendo ele significante ou não) (Tabela 4).

Foi testado o efeito direto da ocupação, do sexo e do nível de complexidade do trabalho para hesitação vacinal. Estas relações diretas apresentaram cargas baixas, não significantes e houve piora nos índices de ajuste do modelo, indicando a necessidade de exclusão destes caminhos e de avaliação do efeito indireto. Verificaram-se efeitos diretos positivos e significantes entre ocupação $(\mathrm{CP}=0,158$; $\mathrm{p}<0,001)$, nível de complexidade do serviço $(\mathrm{CP}=0,176 ; \mathrm{p}=0,014)$ e sexo $(\mathrm{CP}=0,149 ; \mathrm{p}=0,006)$ para acolhimento, com tamanho de efeitos pequenos. Trabalhadores(as) não assistenciais, da média complexidade e do sexo masculino se sentiram menos acolhidos pelo(a) profissional que administra as vacinas (Tabela 4 ).

$\mathrm{O}$ medo de agulhas $(\mathrm{CP}=0,139 ; \mathrm{p}=0,032)$, com efeito pequeno e a conveniência $(\mathrm{CP}=0,351$; $\mathrm{p}<0,001$ ), com efeito médio, apresentaram efeito direto e significante na menor confiança (Tabela 4). 
Tabela 4

Cargas fatoriais e coeficientes padronizados (CP) e valor de $p$ do modelo de equações estruturais utilizando hesitação vacinal como variável resposta. Bahia, Brasil, 2020

\begin{tabular}{|c|c|c|c|c|c|c|}
\hline \multirow[t]{3}{*}{ Itens/Efeitos diretos } & \multicolumn{6}{|c|}{ Modelos de mensuração } \\
\hline & \multicolumn{2}{|c|}{ Modelo 1} & \multicolumn{2}{|c|}{ Modelo 2} & \multicolumn{2}{|c|}{ Modelo 3} \\
\hline & $\mathbf{C P}$ & Valor de $p$ & CP & Valor de $p$ & CP & Valor de $p$ \\
\hline \multicolumn{7}{|l|}{ Confiança } \\
\hline C1 & 0,460 & $<0,001$ & 0,448 & $<0,001$ & 0,436 & $<0,001$ \\
\hline $\mathrm{C} 2$ & 0,625 & $<0,001$ & 0,621 & $<0,001$ & 0,636 & $<0,001$ \\
\hline C3 & 0,933 & $<0,001$ & 0,955 & $<0,001$ & 0,970 & $<0,001$ \\
\hline $\mathrm{C} 4$ & 0,853 & $<0,001$ & 0,814 & $<0,001$ & 0,805 & $<0,001$ \\
\hline $\mathrm{C} 5$ & 0,743 & $<0,001$ & 0,774 & $<0,001$ & 0,757 & $<0,001$ \\
\hline \multicolumn{7}{|l|}{ Conveniência } \\
\hline V1 & 0,667 & $<0,001$ & 0,732 & $<0,001$ & & \\
\hline V2 & 0,680 & $<0,001$ & 0,736 & $<0,001$ & & \\
\hline V3 & 0,305 & $<0,001$ & & & & \\
\hline V4 & 0,348 & $<0,001$ & 0,282 & $<0,001$ & & \\
\hline \multicolumn{7}{|l|}{ Complacência } \\
\hline L1 & 0,194 & 0,001 & & & & \\
\hline L2 & 0,935 & $<0,001$ & & & & \\
\hline L3 & 0,389 & $<0,001$ & & & & \\
\hline \multirow[t]{2}{*}{ Itens/Efeitos diretos } & \multicolumn{2}{|c|}{ Modelo de equação estrutural } & & & & \\
\hline & $\mathbf{C P}$ & Valor de $p$ & & & & \\
\hline \multicolumn{7}{|l|}{ Confiança (on) } \\
\hline Acolhimento & 0,438 & $<0,001$ & & & & \\
\hline Medo de agulhas & 0,139 & 0,032 & & & & \\
\hline História de reação adversa & 0,122 & 0,068 & & & & \\
\hline Conveniência & 0,351 & $<0,001$ & & & & \\
\hline Complacência & 0,167 & 0,004 & & & & \\
\hline \multicolumn{7}{|l|}{ Conveniência (on) } \\
\hline Acolhimento & 0,404 & $<0,001$ & & & & \\
\hline \multicolumn{7}{|l|}{ Complacência (on) } \\
\hline Acolhimento & 0,385 & $<0,001$ & & & & \\
\hline História de reação adversa & 0,168 & $<0,001$ & & & & \\
\hline \multicolumn{7}{|l|}{ Hesitação (on) } \\
\hline Confiança & 0,261 & 0,044 & & & & \\
\hline \multicolumn{7}{|l|}{ Hesitação (on) } \\
\hline Complacência & 0,256 & $<0,001$ & & & & \\
\hline Conveniência & $-0,079$ & 0,249 & & & & \\
\hline Medo de agulhas & 0,063 & 0,216 & & & & \\
\hline História de reação adversa & $-0,066$ & 0,190 & & & & \\
\hline \multicolumn{7}{|l|}{ Acolhimento (on) } \\
\hline Ocupação & 0,158 & $<0,001$ & & & & \\
\hline Atenção primária/Média complexidade & 0,176 & 0,014 & & & & \\
\hline Sexo & 0,149 & 0,006 & & & & \\
\hline
\end{tabular}

C1: Já aconteceu algo na sua vida ou comunidade que fez com que você deixasse de acreditar nas vacinas; C2: acredita que o governo fornece a melhor vacina; C3: confia em informações sobre vacinas; C4: segurança em novas vacinas; C5: confia nos profissionais; L1: Trabalhar com muitas pessoas todo dia aumenta minhas chances de pegar gripe; L2: Eu tenho muito a ganhar ao me vacinar contra gripe; L3: Para me vacinar contra gripe, eu precisaria abrir mão de minhas concepções sobre utilidade, benefícios e riscos da vacinação; V1: acesso a campanhas de vacina; V2: recebe informações sobre riscos/benefícios; V3: campanha de vacinação no meu trabalho; V4: acesso aos meios de informação sobre vacinas. 
Figura 2

Modelo de equação estrutural com efeitos diretos para hesitação vacinal entre trabalhadores da atenção primária e média complexidade de saúde. Santo Antônio de Jesus, Bahia, Brasil, 2020.

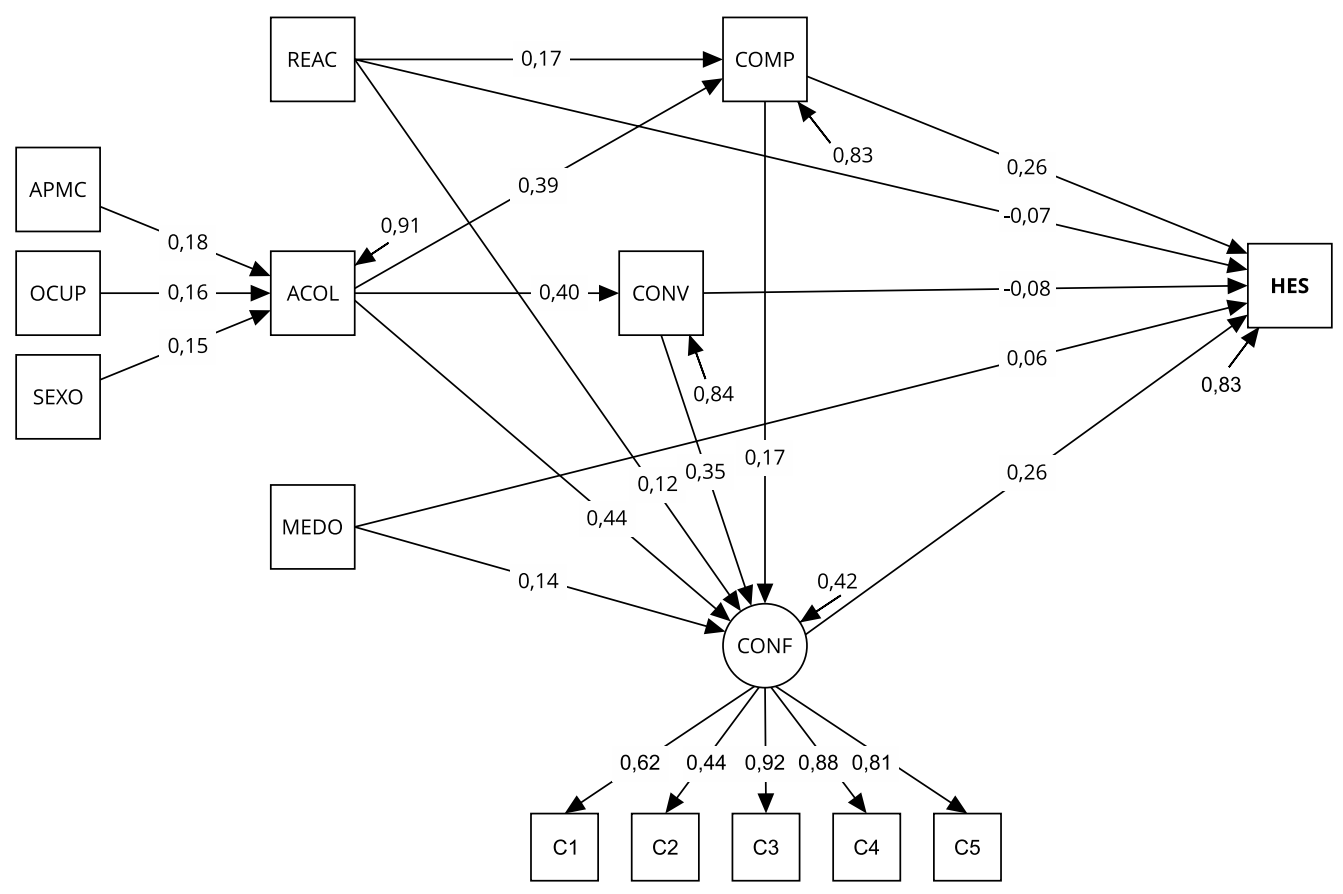

ACOL: acolhimento; APMC: atenção primária e média complexidade; COMP: complacência; CONF: confiança; CONV: conveniência; HES: hesitação; OCUP: ocupação; REAC: reação.

\section{Discussão}

Os achados fornecem evidências de que a menor confiança e a maior complacência estão associadas a maior hesitação vacinal para influenza e que o efeito de conveniência sobre a hesitação foi mediado pelo nível de confiança.

O acolhimento é importante mediador da relação entre sexo, ocupação e nível de complexidade do trabalho com a hesitação vacinal. Entre os componentes do modelo 3Cs existe uma determinação direta à hesitação e, simultaneamente, existe associação entre eles. São, desta forma, importantes mediadores do efeito entre eles, mas também dos outros fatores, a exemplo do acolhimento, sexo e natureza do trabalho desenvolvido.

O modelo estrutural composto pelas dimensões dos 3Cs e características sociais, ocupacionais e de experiências prévias relacionadas à vacinação, revelou boa qualidade de ajuste, sugerindo que, na população de trabalhadores(as), o modelo 3Cs é bom estimador para hesitação vacinal.

A complacência e a confiança associaram-se diretamente com a hesitação. O papel dessas duas dimensões, mais evidente que o da conveniência, pode ser explicado, pelas diferentes formas de acesso às vacinas em todo o mundo. As barreiras relacionadas à conveniência incluem custos relacionados às vacinas e experiências pessoais ou comunitárias de pobreza, no caso dos países que não possuem vacinação gratuita 41 . No caso de acesso gratuito numa rede de serviços abrangente como é o Sistema Único de Saúde (SUS) no Brasil, as barreiras com relação a essa dimensão são reduzidas; logo, a sua relevância na hesitação também decresce. 
Menor confiança foi o fator mais fortemente associado à hesitação. A baixa confiança sobre as vacinas está frequentemente relacionada à hesitação vacinal entre adultos mais velhos, idosos, gestantes, adolescentes e profissionais de saúde 11,42,43. A confiança é um termo que cobre uma diversidade de fatores que afetam as decisões de aceitação da vacinação 27 . A desconfiança entre profissionais de saúde é aumentada à medida que se deparam com restrições de tempo, aumento da carga de trabalho ou mesmo devido a informações inadequadas ou falta de treinamento 44.

$\mathrm{O}$ medo de agulhas esteve associado à menor confiança e à hesitação vacinal. Embora a história de reação vacinal não tenha sido diretamente associada com a hesitação vacinal, mostrou-se associada à maior complacência, isto é, à menor percepção do risco de doenças imunopreveníveis e da utilidade das vacinas. Revisão sistemática identificou que o medo de efeitos adversos é um fator decisivo para a recusa vacinal 45 . Similarmente, no Peru, entre indivíduos adultos que não foram vacinados contra a gripe no ano anterior, "ter medo da vacinação e de seus efeitos" foi a barreira mais citada 46. Assim, registra-se consistência de nossos resultados com achados de estudos de outras populações.

Apesar de atuarem no setor de saúde, trabalhadores(as) da saúde também possuem diferentes níveis de conhecimento relacionados à doença, como a distinção entre resfriado comum e gripe, e equívocos sobre a imunização contra influenza, como a possibilidade de desenvolver sintomas da doença pós-vacinação 47 ou mesmo efeitos colaterais 41 .

Nessa perspectiva, vacinadores(as) possuem papel fundamental para a manutenção da confiança nas vacinas, pois são considerados fonte confiável de informações sobre isto 48. O item "Você confia em informações que os profissionais lhe repassam sobre vacinação?” foi o que apresentou carga mais elevada no construto confiança. Desse modo, acesso à atualização contínua e a informações sobre precauções e eventos adversos relacionados à vacina contra influenza podem potencializar a confiança dos trabalhadores da saúde e diminuir a hesitação em vacinar. São, assim, medidas que podem reduzir as barreiras à vacinação.

Esse aspecto é reforçado em revisão sistemática que evidenciou que as taxas de cobertura de vacinação em vigor são mais baixas do que as taxas regionais regulares estabelecidas pela Organização Pan-Americana da Saúde (OPAS) entre profissionais de saúde. Assim, conclui-se que níveis mais elevados de educação e confiança nos profissionais de saúde favorecem a aceitação às vacinas e que existem desafios relacionados com conhecimento e treinamento dos profissionais de saúde, especificamente sobre vacinas 45 . Portanto, o preparo e atualização do pessoal responsável pela vacinação despontam como elementos cruciais.

Vacinação autorrelatada tem sido maior entre aqueles que atribuem valor à aceitação da vacina 46. Neste estudo, a complacência foi um determinante para hesitação, representada pelo item: "Eu tenho muito a ganhar ao me vacinar contra gripe", ou seja, profissionais que percebem benefícios em receber a vacinação contra influenza tendem a ser menos complacentes.

Revisão sistemática identificou que, entre profissionais de saúde, os grupos que apresentaram menor captação para vacinação pandêmica foram aqueles(as) que não estavam diretamente vinculados às atividades assistenciais 7 . As percepções de risco são altamente influentes para aceitação da vacina. Esta percepção pode depender da ocupação do indivíduo. Ausência ou falhas na percepção dos riscos a que estão expostos durante o trabalho têm sido relatadas entre trabalhadores que não prestam diretamente cuidados à saúde, fazendo com que subestimem os riscos vivenciados 9,26.

Registra-se ainda, entre trabalhadores(as), a crença de que não correm o risco de contrair a gripe e de que, se contraírem, não terão complicações decorrentes da doença, por se considerarem saudáveis 10,47. Essa subestimação da magnitude e gravidade da doença parece equivocada, uma vez que estudo recente de modelagem estimou que a infecção anual pela gripe está associada a aproximadamente nove a cada 100 mil mortes em todo o mundo 4 .

São incipientes os estudos conduzidos com trabalhadores(a) da média complexidade, mas similarmente aos profissionais dos serviços hospitalares, sabe-se que esses indivíduos podem não estar cientes da necessidade de se vacinar contra a gripe 49 ou ter menor acesso à vacinação 13 ou mesmo às campanhas, considerando a distância do trabalho dos pontos de vacinação 49 .

As barreiras de acesso à vacinação entre esses(as) trabalhadores(as) de serviços assistenciais da média complexidade precisam ser analisadas de forma holística, considerando inclusive acesso e maior visibilidade às campanhas de vacinação em conjunto com atividades de formação específica sobre o tema 50. 
Estudo no Canadá identificou que a disposição em receber uma nova vacina foi consistentemente associada com a percepção de segurança da vacina, mediante o suporte profissional recebido durante a vacinação 51 . O apoio social e de colegas também foram considerados fatores que contribuem para a confiança e aumentam a possibilidade do(a) trabalhador(a) recomendar vacinas 52 .

$\mathrm{O}$ acolhimento em sala de vacina constitui um dos dispositivos cruciais para adesão aos imunizantes. A sala de vacina é espaço onde se potencializa a confiança e se assegura a conveniência, por meio do acesso às informações adequadas e claras sobre os imunizantes disponíveis e o local propício para se trabalhar percepção de risco, principalmente para trabalhadores(as) de saúde. Mesmo em países de alta renda com vacinação amparada em recursos significativos aos programas de imunização, se a comunicação for inadequada, a hesitação se potencializa 53.

A pandemia decorrente da COVID-19 e seus efeitos poderão aumentar a percepção de risco para influenza e a visão da imunização como norma social, diminuindo, assim, a complacência para influenza 24. Os desafios impostos pela recusa desta vacina estimulam o interesse em compreender as atitudes do público para as demais vacinas, considerando a possibilidade de dupla epidemia iminente da influenza-COVID-19 54. A desinformação ganha destaque como barreira que dificulta o alcance de imunidade coletiva por meio da vacina e isso pode afetar a recepção de outras vacinas 22,54.

Este estudo tem caráter inovador ao abordar, no Brasil, a hesitação vacinal entre trabalhadores(as) dos serviços de saúde, utilizando tanto o modelo 3C quanto técnicas avançadas de análise de dados, com a finalidade de propiciar melhor compreensão de como os 3Cs estão relacionados entre si e as influências diretas e indiretas que exercem sobre a hesitação à vacina contra influenza. Esse caráter inédito implicou dificuldades na comparação dos resultados obtidos. Na literatura, não estavam disponíveis dados para uma análise comparativa mais sólida.

Cabe destacar outras limitações. Apesar da participação de 453 trabalhadores, o n amostral limitou o número de variáveis utilizado no SEM. Contudo, segundo Hair Jr. et al. 37, para o processamento de modelagem com equações estruturais, amostras maiores que 200 são consideradas grandes, contanto que tenha minimamente entre 5 e 10 respondentes para cada parâmetro estimado - condição atendida neste estudo. Também foram incluídas todas as outras profissões que trabalhavam no setor saúde, permitindo avaliação abrangente das situações de interesse.

A dimensão complacência apresentou baixas cargas fatoriais para operacionalização como variável latente. Apenas o L2, isoladamente, permitiu a mensuração por meio de uma variável diretamente observada.

A dimensão conveniência incluiu itens que possivelmente não exploram aspectos que afetam, de diferentes maneiras, o acesso às vacinas contra a influenza e, por isso, foi utilizada como variável diretamente observada.

Recentemente foi proposto o modelo $5 \mathrm{C}$ de determinantes da hesitação vacinal, que amplia o modelo 3C 16, incorporando as dimensões do "cálculo" (Calculation - engajamento na busca de informações) e "responsabilidade coletiva"(Collective responsibility - disposição para proteger os outros). Neste estudo, não foi possível explorar o eventual papel dessas duas novas dimensões, já que o seu planejamento ocorreu simultaneamente à proposição desse novo modelo, inviabilizando o processo de adaptação cultural da escala em tempo hábil.

\section{Considerações}

O principal achado deste estudo foi o suporte à hipótese de que a confiança e aspectos relacionados à complacência associaram-se ao maior escore de hesitação vacinal, indicando que fatores relacionados a essas duas dimensões produziram prejuízos na aceitação desta vacina e devem ser considerados no desenvolvimento de estratégias para buscar maior adesão da população à vacinação contra influenza e, eventualmente, outras infecções. 


\section{Colaboradores}

F. O. Souza e T. M. Araújo contribuíram com a concepção e desenho do estudo; coleta, análise e interpretação dos dados; e redação do artigo. G. L. Werneck contribuiu com a análise e interpretação dos dados; redação do artigo; e revisão crítica. P. S. Pinho contribuiu com a concepção e desenho do estudo e coleta, análise e interpretação dos dados. J. R. B. Teixeira e I. Lua contribuíram com a análise e interpretação dos dados e redação do artigo. Todos(as) os(as) autores(as) aprovaram a versão final a ser publicada.

\section{Informações adicionais}

ORCID: Fernanda de Oliveira Souza (0000-00033573-9801); Guilherme Loureiro Werneck (00000003-1169-1436); Paloma de Sousa Pinho (00000001-6402-0869); Jules Ramon Brito Teixeira (0000-0002-8443-7810); Iracema Lua (0000-00025760-5073); Tânia Maria de Araújo (0000-00032766-7799).

\section{Agradecimentos}

Ao Conselho Nacional de Desenvolvimento Científico e Tecnológico (CNPq; processo no 440691/2016-8).

\section{Referências}

1. Ministério da Saúde. Plano de contingência para resposta às emergências em saúde pública. http://www.saude.ba.gov.br/wp-content/ uploads/2019/05/2018-Plano-de-Conting\% C3\%AAncia-para-Sazonalidade-e-Epidemiasde-Influenza.pdf (acessado em 14/Abr/2021).

2. Ministério da Saúde. Instrução Normativa. Calendário Nacional de Vacinação. Adaptação: Núcleo de Imunizações/DVE/CEVS/ SES. https://www.cevs.rs.gov.br/upload/arqui vos/202010/05141508-instrucao-normativacalendario-nacional-de-imunizacoes-2020.pdf (acessado em 14/Abr/2021).

3. World Health Organization. Seasonal influenza is an acute respiratory infection caused by influenza viruses which circulate in all parts of the world. https://www.who.int/en/news$\mathrm{room} /$ fact-sheets/detail/influenza-(seasonal) (acessado em 19/Jun/2021).

4. Iuliano AD, Roguski KM, Chang HH, Muscatello DJ, Palekar R, Tempia S, et al. Estimates of global seasonal influenza-associated respiratory mortality: a modelling study. Lancet 2018; 391:1285-300.

5. Palekar RS, Rolfes MA, Arriola CS, Acosta BO, Guidos PA, Vargas XB, et al. Burden of influenza-associated respiratory hospitalizations in the Americas, 2010-2015. PLoS One 2019; 14:e0221479.

6. Squeri R, Riso R, Facciolà A, Genovese C, Palamara MAR, Ceccio C, et al. Management of two influenza vaccination campaign in health care workers of a university hospital in the south Italy. Ann Ig 2017; 29:223-31.

7. Schmid P, Rauber D, Betsch C, Lidolt G, Denker M-L. Barriers of influenza vaccination intention and behavior: a systematic review of influenza vaccine hesitancy, 2005 - 2016. PLoS One 2017; 12:e0170550.

8. Gentile A, Paget J, Bellei N, Torres JP, Vazquez $\mathrm{C}$, Laguna-Torres VA, et al. Influenza in Latin America: a report from the Global Influenza Initiative (GII). Vaccine 2019; 37:2670-8

9. Alshammari TM, AlFehaid LS, AlFraih JK, Aljadhey HS. Health care professionals' awareness of, knowledge about and attitude to influenza vaccination. Vaccine 2014; 32:5957-61.

10. Vírseda S, Restrepo MA, Arranz E, MagánTapia P, Fernández-Ruiz M, de la Cámara AG, et al. Seasonal and pandemic A (H1N1) 2009 influenza vaccination coverage and attitudes among health-care workers in a Spanish University Hospital. Vaccine 2010; 28:4751-7.

11. Neufeind J, Betsch C, Habersaat KB, Eckardt M, Schmid P, Wichmann O. Barriers and drivers to adult vaccination among family physicians: insights for tailoring the immunization program in Germany. Vaccine 2020; 38:4252-62.

12. Albano L, Matuozzo A, Marinelli P, Di Giuseppe G. Knowledge, attitudes and behaviour of hospital health-care workers regarding influenza A/H1N1: a cross sectional survey. BMC Infect Dis 2014; 14:208. 
13. Arghittu A, Dettori M, Azara A, Gentili D, Serra A, Contu B, et al. Flu vaccination attitudes, behaviours, and knowledge among health workers. Int J Environ Res Public Health 2020; 17:3185.

14. Souza TP, Lobão WM, Santos CAST, Almeida MCC, Moreira Júnior ED. Fatores associados à aceitação da vacina influenza entre trabalhadores de saúde: conhecimento, atitude e prática. Ciênc Saúde Colet 2019; 24:3147-58.

15. MacDonald NE; SAGE Working Group on Vaccine Hesitancy. Vaccine hesitancy: definition, scope and determinants. Vaccine 2015; 33:4161-4.

16. Betsch C, Schmid P, Heinemeier D, Korn L, Holtmann C, Böhm R. Beyond confidence: development of a measure assessing the $5 \mathrm{C}$ psychological antecedents of vaccination. PLoS One 2018; 13:e208601.

17. Jarrett C, Wilson R, O'Leary M, Eckersberger E, Larson HJ; SAGE Working Group on Vaccine Hesitancy. Strategies for addressing vaccine hesitancy: a systematic review. Vaccine 2015; 33:4180-90.

18. Piccirillo B, Gaeta T. Survey on use of and attitudes toward influenza vaccination among emergency department staff in a New York metropolitan hospital. Infect Control Hosp Epidemiol 2006; 2:618-22.

19. Montserrat-Capdevila J, Godoy P, Marsal JR, Barbé-Illa F. Factores asociados a recibir la vacunación antigripal en profesionales de atención primaria. Gac Sanit 2015; 29:383-6.

20. Karafillakis E, Dinca I, Apfel F, Cecconi S, Würz A, Takacs J, et al. Vaccine hesitancy among healthcare workers in Europe: a qualitative study. Vaccine 2016; 34:5013-20.

21. Zarobkiewicz MK, Zimecka A, Zuzak T, Cieślak D, Roliński J, Grywalska E. Vaccination among Polish university students: knowledge, beliefs and anti-vaccination attitudes. Hum Vaccin Immunother 2017; 13:2654-8.

22. Zhang Y, Fisk RJ. Barriers to vaccination for coronavirus disease 2019 (COVID-19) control: experience from the United States. Glob Health J 2021; 5:51-5.

23. World Health Organization. Ten threats to global health in 2019. https://www.who.int/ news-room/spotlight/ten-threats-to-globalhealth-in-2019 (acessado em 14/Abr/2021).

24. González-Block MÁ, Gutiérrez-Calderón E, Pelcastre-Villafuerte BE, Arroyo-Laguna J, Comes Y, Crocco P, et al. Influenza vaccination hesitancy in five countries of South America. Confidence, complacency and convenience as determinants of immunization rates. PLoS One 2020; 15:e0243833.

25. Verger P, Fressard L, Collange F, Gautier A, Jestin C, Launay $\mathrm{O}$, et al. Vaccine hesitancy among general practitioners and its determinants during controversies: a national crosssectional survey in France. EBioMedicine $2015 ; 2: 891-7$.
26. Araújo TM, Souza FO, Pinho PS. Vacinação e fatores associados entre trabalhadores da saúde. Cad Saúde Pública 2019; 35:e00169618.

27. Strategic Advisory Group of Experts. Report of the SAGE Working Group on vaccine hesitancy. https://www.who.int/immuniza tion/sage/meetings/2014/octobe/1_Report_ WORKING_GROUP_vaccine_hesitancy_ final.pdf (acessado em 14/Jun/2021).

28. Shahrabani S, Benzion U, Yom Din G. Factors affecting nurses' decision to get the flu vaccine. Eur J Health Econ 2009; 10:227-31.

29. Blue CL, Valley JM. Predictors of influenza vaccine. Acceptance among healthy adult workers. AAOHN J 2002; 50:227-33.

30. Neves CR, Codeço CT, Luz PM, Garcia LMT. Preditores de aceitação da vacina contra influenza: tradução para o português e validação de um questionário. Cad Saúde Pública 2020; 36 Suppl 2:e00211518.

31. Gefen D, Straub D, Boudreau M-C. Structural equation modeling and regression: guidelines for research practice. Communications of the Association for Information Systems 2000; 4:7.

32. Pugesek BH, Tomer A, von Eye A, organizadores. Structural equation modeling: applications in ecological and evolutionary biology. Cambridge/New York: Cambridge University Press; 2003.

33. Amorim LDAF, Fiaccone RL, Santos CAST, Santos TN, Moraes LTLP, Oliveira NF, et al. Structural equation modeling in epidemiology. Cad Saúde Pública 2010; 26:2251-62.

34. Amorim LDAF, Fiaccone R, Santos C, Moraes L, Oliveira N, Oliveira S, et al. Modelagem com equações estruturais: princípios básicos e aplicações. http://repositorio.ufba.br/ri/hand le/ri/17684 (acessado em 14/abr/2021).

35. Curado MAS, Teles J, Marôco J. Analysis of variables that are not directly observable: influence on decision-making during the research process. Rev Esc Enferm USP 2014; 48:146-52.

36. Marsh HW, Muthén B, Asparouhov T, Lüdtke O, Robitzsch A, Morin AJS, et al. Exploratory structural equation modeling, integrating CFA and EFA: application to students' evaluations of university teaching. Struct Equ Modeling 2009; 16:439-76.

37. Hair Jr. JF, Black WC, Babin BJ, Anderson RE, Tatham RL. Análise multivariada de dados. 6a Ed. Porto Alegre: Bookman; 2009.

38. Reichenheim ME, Hökerberg YHM, Moraes CL. Assessing construct structural validity of epidemiological measurement tools: a seven-step roadmap. Cad Saúde Pública 2014; 30:927-39.

39. Kline R. Principles and practice of structural equation modeling. New York: The Guilford Press; 2015. 
40. Brown TA. Methodology in the social sciences. Confirmatory factor analysis for applied research. 2a Ed. New York: The Guilford Press; 2015.

41. Quinn S, Jamison A, Musa D, Hilyard K, Freimuth V. Exploring the continuum of vaccine hesitancy between African American and white adults: results of a qualitative study. PLoS Curr 2016; 8:ecurrents.outbreaks.3e4a5 ea39d8620494e2a2c874a3c4201.

42. Alici DE, Sayiner A, Unal S. Barriers to adult immunization and solutions: personalized approaches. Hum Vaccin Immunother 2016; 13:213-5.

43. Wang LD, Lam WW, Wu JT, Liao Q, Fielding R. Chinese immigrant parents' vaccination decision making for children: a qualitative analysis. BMC Public Health 2014; 14:133.

44. Oduwole EO, Pienaar ED, Mahomed H, Wiysonge CS. Current tools available for investigating vaccine hesitancy: a scoping review protocol. BMJ Open 2019; 9:e033245.

45. Guzman-Holst A, DeAntonio R, Prado-Cohrs D, Juliao P. Barriers to vaccination in Latin America: a systematic literature review. Vaccine 2020; 38:470-81.

46. Reinders S, Romero C, Carcamo C, Tinoco Y, Valderrama M, La Rosa S, et al. A community-based survey on influenza and vaccination knowledge, perceptions and practices in Peru. Vaccine 2020; 38:1194-201.

47. Hwang SW, Lim HB. Barriers and motivators of influenza vaccination uptake among primary healthcare workers in Singapore. Proceedings of Singapore Healthcare 2014; 23:126-33.

48. Mizuta AH, Succi GM, Montalli VAM, Succi RCM. Percepções acerca da importância das vacinas e da recusa vacinal numa escola de medicina. Rev Paul Pediatr 2019; 37:34-40.
49. Bazán M, Villacorta E, Barbagelatta G, Jimenez MM, Goya C, Bartolini RM, et al. Health workers' attitudes, perceptions and knowledge of influenza immunization in Lima, Peru: a mixed methods study. Vaccine 2017; 35:2930-6.

50. Wilson R, Zaytseva A, Bocquier A, Nokri A, Fressard L, Chamboredon P, et al. Vaccine hesitancy and self-vaccination behaviors among nurses in southeastern France. Vaccine 2020; 38:1144-51.

51. Gilca V, Boulianne N, Dubé E, Sauvageau C, Ouakki M. Attitudes of nurses toward current and proposed vaccines for public programs: a questionnaire survey. Int J Nurs Stud 2009; 46:1219-35.

52. Duval B, Gilca V, Boulianne N, Pielak K, Halperin B, Simpson MA, et al. Cervical cancer prevention by vaccination: nurses' knowledge, attitudes and intentions. J Adv Nurs 2009; 65:499-508.

53. Paterson P, Meurice F, Stanberry LR, Glismann S, Rosenthal SL, Larson HJ. Vaccine hesitancy and healthcare providers. Vaccine 2016; 34:6700-6.

54. Ferdinand KC, Nedunchezhian S, Reddy TK. The COVID-19 and influenza "twindemic": barriers to influenza vaccination and potential acceptance of SARS-CoV-2 vaccination in African Americans. J Natl Med Assoc 2020; 112:681-7. 
Abstract

The World Health Organization acknowledges vaccine hesitancy as one of the ten most serious global health threats. The study investigated the association between confidence, convenience, and complacency and influenza vaccine hesitancy among male and female health workers. The study included 453 workers in primary and mediumcomplexity health services in a medium-sized city in the state of Bahia, Brazil. Vaccine-hesitant individuals were defined as those who had not received an influenza vaccine in 2019. Structural equation models were used to assess interrelations between target predictive variables and vaccine hesitancy. One-fourth of the workers (25.4\%) hesitated to be vaccinated for influenza. Lower confidence (standardized coefficient $-S C=0.261$; $p=0.044)$ and higher complacency $(S C=0.256$; $p<0.001)$ were significantly associated with vaccine hesitancy. Convenience was not associated with vaccine hesitancy. Workers not involved in patient care, workers in medium-complexity services, and male workers reported less receptiveness from the health professional administering the vaccines. Fear of needles was associated with both lower confidence and greater vaccine hesitancy. History of vaccine reactions was not associated directly with vaccine hesitancy, but it was associated with greater complacency, that is, less perception of risk from vaccine-preventable diseases. Since annual influenza vaccination is recommended, influenza vaccine hesitancy can increase the burden of this disease in the population. The data back the hypothesis that factors related to confidence and complacency produce harms in this vaccine's acceptance and should be considered in the development of strategies and actions for greater adherence to vaccination.

Vaccination; Health Personnel; Vaccination Refusal

\section{Resumen}

La Organización Mundial de la Salud reconoce la vacilación en la vacunación como una de las diez mayores amenazas para la salud pública en el mundo. Este estudio investigó la asociación entre confianza, conveniencia y complacencia, así como la indecisión para vacunarse contra la gripe entre trabajadores/as del sector salud. Se incluyeron a 453 trabajadores/as de servicios de atención primaria y media complejidad de una ciudad de tamaño medio en Bahía, Brasil. Se consideraron vacilantes para vacunarse a aquellos que no recibieron una vacuna contra la gripe en 2019. Se utilizaron los modelos de ecuaciones estructurales para evaluar interrelaciones entre variables predictoras de interés y vacilación en vacunarse. Un cuarto de los/as trabajadores/as $(25,4 \%) d u d a-$ ron en si vacunarse contra la gripe. Menor confianza (coeficiente estandarizado - $C E=0,261$; $p=0,044)$ y mayor complacencia $(C E=0,256$; $p<0,001)$ se asociaron significativamente a la vacilación en la vacunación. La conveniencia no estuvo asociada a la vacilación en la vacunación. Los trabajadores/as no asistenciales, de media complejidad y de sexo masculino informaron de una menor acogida por parte del profesional que administraba las vacunas. El miedo a las agujas se asoció a una menor confianza y a la vacilación en la vacunación. Un historial de reacción a la vacunación no estuvo asociado directamente con la vacilación en la vacunación, pero se asoció a una mayor complacencia, esto es, menor percepción del riesgo de enfermedades inmunoprevenibles. Al tratarse de una vacuna recomendada anualmente, la vacilación en la vacunación para la gripe puede contribuir a aumentar la carga de enfermedad en la población. Los datos sostienen la hipótesis de que factores relacionados con la confianza y la complacencia producen prejuicios en la aceptación de esta vacuna, debiendo ser considerados en el desarrollo de estrategias y acciones para una mayor adhesión a la vacunación.

Vacunación; Personal de Salud; Negativa a la Vacunación
Recebido em 20/Abr/2021

Versão final reapresentada em 26/Jul/2021

Aprovado em 29/Jul/2021 